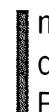
$m$ letzten mAGAzin (№ 5) ist bereits ausführlich auf das Thema Tourismus, auf die Institutionen im Bereich Tourismus und die Bedeutung im wirtschaftlichen Kontext eingangen worden. Ich möchte mit diesem Artikel ein wenig mit Überlegungen zur Unterrichtspraxis und mit Praxisbeispielen beitragen., die ich im Laufe meiner langjährigen Erfahrung in im Hotelbetrieb, also in situ, gegebenen Intensivkursen habe sammeln können.

Grundsätzlich sei darauf hingewiesen, dass die Fremdsprachendidaktik in einer nicht deutschsprachigen Umgebung mit anderen Problemen zu tun hat als in einer deutschsprachigen und zusätzlich die Bedingungen des Tourismus im "Reiseempfängerland"
Dieser pragmatisch-funktionale und kommunikative Ansatz (Neuner, Hunfeld 1997:85) allein genügt allerdings nicht, wenn man das Deutschlernen im Tourismusbereich nicht nur darauf reduzieren will, dass ein Rezeptionist oder ein Kellner den adäquaten Satz ausspricht, um den deutschen Touristen korrekt bedienen zu können. Der funktionale Ansatz ist sinnvoll, um die Umstände der Zielgruppe zu verstehen und den Unterricht nach ihren $\begin{array}{cl}\text { Neqociado de Relaciones } & \text { Notwendigkeiten aufbauen zu kön- } \\ \text { INTERnacionales de la } & \text { nen. Es besteht allerdings die Gefahr } \\ \text { Universidad de Cádiz } & \text { die Lerngruppe als Personen auf eine } \\ & \text { Funktion zu reduzieren: "Personen } \\ \text { tun hat als in lernen Deutsch, um Deutsche besser bedienen zu kön- }\end{array}$ $\begin{array}{cl}\text { Negociado de Relaciones } & \text { Notwendigkeiten aufbauen zu kön- } \\ \text { Internacionales de la } & \text { nen. Es besteht allerdings die Gefahr } \\ \text { Universidad de Cádiz } & \text { die Lerngruppe als Personen auf eine } \\ & \text { Funktion zu reduzieren: "Personen } \\ \text { tun hat als in lernen Deutsch, um Deutsche besser bedienen zu kön- }\end{array}$ $\begin{array}{cc}\text { Negociado de Relaciones } & \text { Notwendigkeiten aufbauen zu kön- } \\ \text { Internacionales de la } & \text { nen. Es besteht allerdings die Gefahr } \\ \text { Universidad de Cádiz } & \text { die Lerngruppe als Personen auf eine } \\ & \text { Funktion zu reduzieren: "Personen } \\ \text { tun hat als in lernen Deutsch, um Deutsche besser bedienen zu kön- }\end{array}$ $\begin{array}{cl}\text { Negociado de Relaciones } & \text { Notwendigkeiten aufbauen zu kön- } \\ \text { Internacionales de la } & \text { nen. Es besteht allerdings die Gefahr } \\ \text { Universidad de Cádiz } & \text { die Lerngruppe als Personen auf eine } \\ & \text { Funktion zu reduzieren: "Personen } \\ \text { tun hat als in lernen Deutsch, um Deutsche besser bedienen zu kön- }\end{array}$ nen!!!".

\title{
DEUTSCH ALS FREMDSPRACHE IM BEREICH TOURISMUS
}

L.Moholy-Nagy, anuncio para la serie de los "Libros de la Bauhaus", 1926.

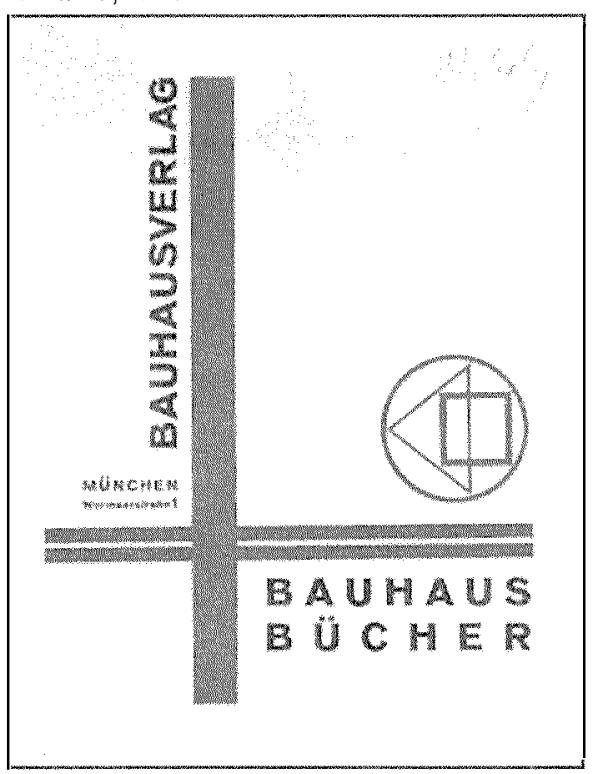

für die Unterrichtspraxis anders sind als ein einem typischen

"Touristensendeland" wie Deutschland.

Deutsch als Fremdsprache im Bereich Toursimus im "Tourimusempfängerland", in unserem Beispiel, Südspanien, erfordert zum Beginn folgende Grundüberlegungen $\mathrm{zu}$ den Motivationen der meisten Lerner, die sich für Deutsch entscheiden.

- Wozu lernt der Schüler diese Sprache?

- Welche fundamentalen

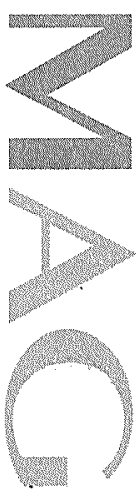

Kommunikationssituationen sind von Bedeutung?

- Welches allgemeine und welches spezifische Vokabular braucht der Lerner?

- Wo und in welchen Kontexten wird er diese Sprache praktisch anwenden?

- Wie kann der Schüler das "Wichtigste" möglichst schnell lernen? (Was ist "das Wichtigste"?)
Deutschlerner sind Mitglieder einer demokratischen (Blanco 1995) und interkulturellen (Krumm 1995) Gesellschaft, die sich ständig verändert und entwickelt. Toursimus bedeutet nicht nur, Touristen irgedwie zu empfangen und zu bedienen, sondern auch die allgemeine Möglichkeit, ein Produkt mit eigenen Resourcen, sei es Landschaft, Sehenswürdigkeiten, Gastronomie zu schaffen und zu verbessern und damit die Wirtschaft, den Arbeitsmarkt und ganz allgemein die Lebensqualität der Region zu verbessern und zu erhalten. Es soll nicht nur bedeuten, daß diese touristischen Resourcen einer Region für einen kurzfristigen und fragwürdigen kommerziellen Gewinn geopfert werden.

Deutsch als Fremdsprache im Fachbereich Tourismus scheint sich auf den ersten Blick nur auf die elementalen Kommunikationsituationen im Hotel- und Gaststättengewerbe zu beziehen, enweitert sich aber auf komplexere Situationen, z. B. Animation, Hotelleitung, Saisonplanung oder allgemein die gesamte Tourismusökonomie (Freyer 1993) und -politik. Sowohl der traditionelle Massentourimus als auch innovativere und ökologische Möglichkeiten, die Resourcen eines Fremdenverkehrsgebietes zu nutzen, können als Themen in Deutsch als Fremdsprache behandelt werden (Scherer 1995). 


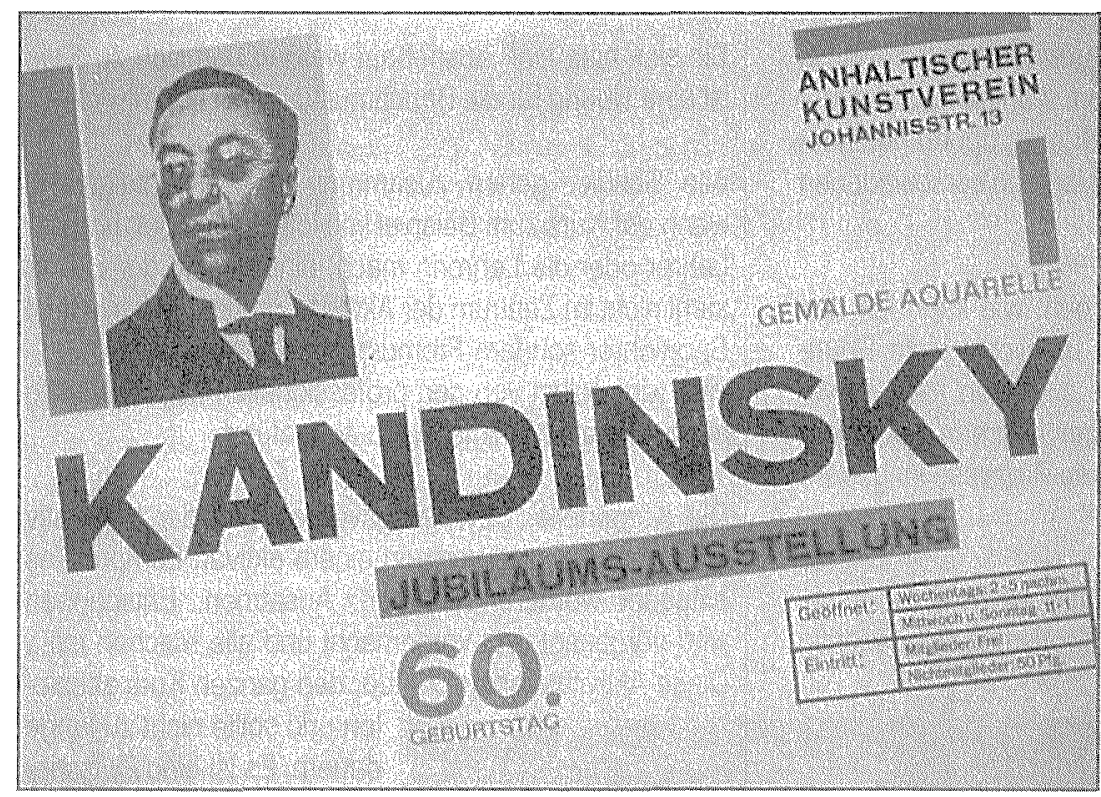

Cartel de Herbert Bayer

Welche allgemein pädagogische und ethische Ziele (Neuner, Hunfeld 1997, 85) können nun unter diesem Gesichtspunkt ins Auge gefasst werden?

Der interkulturelle Anspruch (Neuner, Hunfeld 1997, 106) kann uns da weiterhelfen. Es geht im Bereich Tourismus darum, dem Fremden die eigene Regional- und Landeskultur, die ihm unbekannt und schon deswegen attraktiv ist,. zu vermitteln und näher zu bringen. Es ist sinnvoll, dass die Lerner die Kultur der deutschsprachigen Länder verstehen, um nachvollziehen zu können, was der Fremde an der lokalen Kultur nicht versteht oder welche möglichen Missverständnisse sich dadurch ergeben können.

z. B: Er weiss die Tageseinteilung in Deutschland, und kann auf Grund dieser Kenntnisse den deutschsprachigen Touristen auf Deutsch erklären, dass die Öffnungszeiten der Geschäfte anders sind, und um wieviel Uhr gegessen wird etc. Das Ziel soll dabei nicht sein, dass sich die lokalen Gewohnheiten den Touristen anpassen, sondern dass die lokalen Gewohnheiten dem Fremden verständlich werden und deswegen als besonders attraktiv erscheinen. Man kann eine Siesta schlafen und danach einen Bummel durch die Stadt machen, und man sollte nicht etwa bei grö(ter Hitze im August um 4 Uhr nachmittags durch eine wie ausgestorbene Innenstadt spazierengehen.
Die Lerner im Bereich Tourismus interessieren sich im Allgemeinen zunächst weder für die deutschprachigen Länder, noch haben sie die unmittelbare Absicht, nach Deutschland, Österreich oder in die Schweiz zu reisen oder sich dort länger aufzuhalten.

Die meisten Lerner entscheiden sich für Deutsch, weil sie glauben, wenn sie Deutsch lernen, haben sie mehr Möglichkeiten, einen Arbeitsplatz im Tourismussektor zu finden. Tatsache ist, dass ein großer Prozentsatz an Deutschen einmal im Jahr eine Reise macht. Die beliebtesten Reiseziele sind Spanien, Italien und andere Mittelmeerländer (Freyer 1993, Montaner 1990, Scherer 1995). In diesen Touristenempfängerländern ist Deutsch im allgemeinen nicht die erste, und oft auch nicht die zweite Fremdsprache, weder in der Sekundarstufe noch im berufsbildenden Bereich. Dies könnte sich aber in den Regionen ändern, deren Haupteinnahmequelle der Tourismus ist und die eine große Anzahl von Touristen aus deutschsprachigen Ländern empfangen, da es für alle, die im Fremdenverkehrsbereich und in Fremdenverkehrsgebieten mit vielen deutschsprachigen Touristen arbeiten, gewissermaßen "rentabel" ist, Deutsch zu lernen (Neuner 1998 im mAGAzin Nr. 4 und Interview mit L. Robledo 1998 im mAGAzin Nr. 4).

\section{Erfahrungs- und Lernprozesse}

Mit den Erkenntnissen aus der kognitiven Psychologie (Linke/ Nussbaumer/ Portmann 1991) und den neueren Forschungen des kognitiven und angeborenen Spracherwerbs (Haidl 1999) sollte der Unterricht für die Lerner und mit den Lernern (Contreras) in einem "relevanten Kontext" (Wilson) geschaffen werden, so dass sich unvergessliche Erfahrungen abspielen, und es nicht nur eine große Anstrengung ist, die, wenn unmittelbar danach alles vergessen wird, umsonst war (Pérez Gómez 1995). Über Erfahrungen lernt man und diese Erfahrungen vergisst man meistens nie wieder. Authen- 
tische und unvergessliche Erfahrungen können nur in einem für die Lerner relevanen Kontext stattinden. Nicht alle Aktivitäten sind für alle Lerngruppen geeignet und nicht alle Aktivitäten werden von den verschiedenen Lerngruppen gleich akzeptiert.

Die entscheidenende Frage ist nun, wie kann ich den Unterricht gestalten, damit sich möglichst viele Erfahrungs- und Interaktionsprozesse zwischen allen anwesenden Lernern einschließlich der Lehrerin oder dem Lehrer abspielen? Wie laufen diese Prozesse im gegenseitigen Austausch ab?

In den folgenden Kapiteln möchte ich drei Beispiele vorstellen, die für den Bereich Tourismus sehr wichtig sind:

- Animation: als Beispiel Gymnastik: "Aktive" Lernprozesse

- Gastronomie: Wortschatzarbeit und Textproduktion im relevanten Kontext

- Ausflüge in die regionale Umgebung: Projekt- und Gruppenarbeit, Problemlösung und Lernprozess

\section{Gymnastik im Deutschun- terricht}

Je nach Art des Kurses, Intensivkurse zum Beispiel ist es möglich Gymnastik zu veranstalten. Animateure bieten diese Aktivität in den Hotels an, und es ist auch eine gute Möglichkeit, aus dem normalen UnterrichtsMarcel Breuer, 1922 trott auszubrechen.

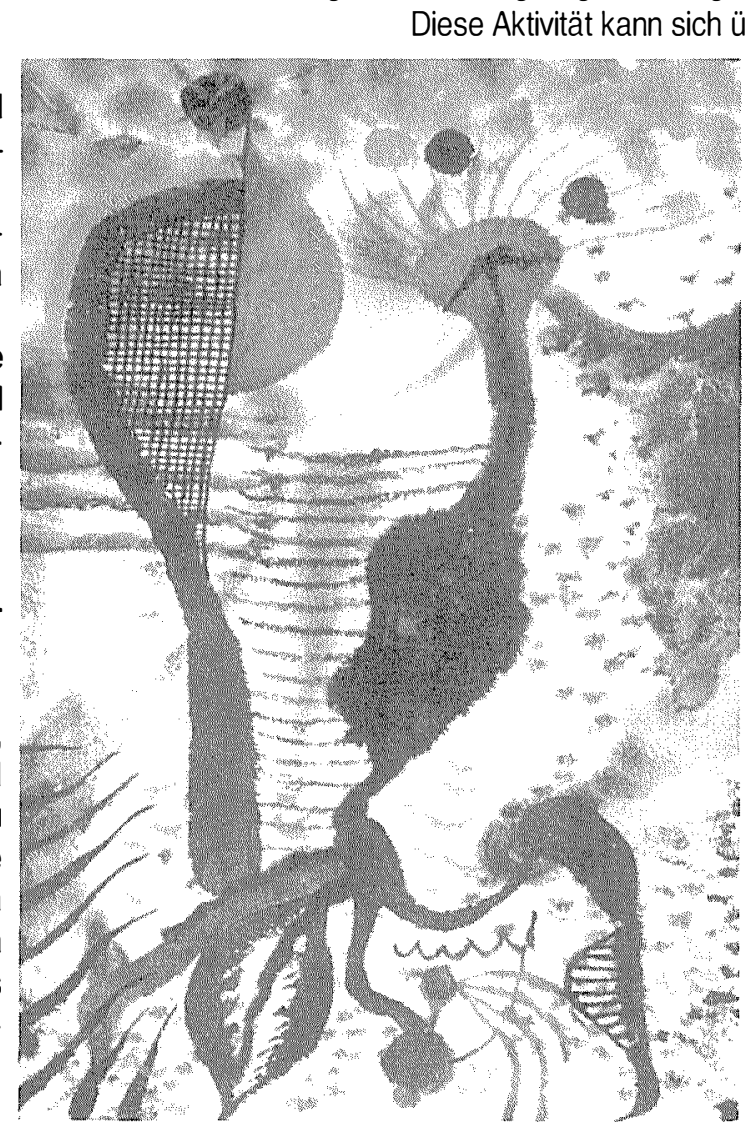

Sie sich bitte hin!" ausgebaut werden und sich zu "Das ist eine Übung für die Bauchmuskeln." weiterentwickeln.

Alle Schüler gehen zusammen in den Hotelgarten, wenn der Kurs zum Beispiel im Hotel stattfindet, und der Lehrer oder die Lehrerin macht einfach mit, der Lehrer steht nicht im Zentrum der Aktivität, da der Lehrer ja kein Sportlehrer sondern Fremdsprachenlehrer ist. Die Aktivität wird selbst von den professionellen oder zukünftig professionellen Animateuren ausgeführt.

Die Körperteile, Bewegungsverben und der Imperativ können auf diese Weise in einem aktiven Lernprozess eingeübt werden: Einatmen, Ausatmen, Entspannen wird vorgesagt und vorgemacht und alle machen mit... Diese Aktivität kann sich über den ganzen Kurs abspieDiese Aktivat kann sich uber den ganzen Kurs abspielen und sollte nicht zu lange
dauern. Es ist eine Zwischenaktivität, die zur Auflockerung des Unterrichts beitragen kann. Der jeweilige Schüler, der "dran" ist, muß am Vortag alle darauf hinweisen, dass sie Turnschuhe und die Sportkleidung nicht vergessen sollen

Zum Begriff des authentischen Textes im Bereich Tourismus im Empfängerland

Die Gymnastik wird von den Schülern selbst veranstaltet, da sie ja die Animateure sind oder es werden möchten. Die ersten Male kann es besonders bei den Anfängern auf Gestik und Vormachen, "1, 2 und 3" und einfach mitmachen basieren und dann aber zu: "Kommt bitte mit!" "Ich mache es vor." "Holt die Matten" "Legen schen Speisekarten sind meir Sersurter haften Deutsch verfasst. Es ist aber sehr wichtig, dass die Schüler von Anfang an lernen, andere Speisekarten oder andere deutsche Textproduktionen des regionalen Tourismus nicht blind zu kopieren, sondern zuerst zu untersuchen, was eigentlich inhaltich und welches Vokabular verwertbar ist (Kleppin 1998). 
Authentische Texte von deutschen Restaurants aus Deutschland wären in diesem Kontext nicht sinnvoll. Es hat nur Sinn, eine Leberknödelsuppe oder ähnliches in der jeweiligen gastronomischen Region zu behandeln. Eine Leberknödelsuppe wird in Spanien gegebenenfalls in einem auf deutsches Essen spezialisiertes Restaurant angeboten. Das ist aber nicht unser Ziel. Unser Ziel ist es, die lokale Gastronomie anzubieten. Das Problem der authentischen Texte aus der Region ist aber, dass sie oft auf Grund der Umstände Mängel aufweisen. Dies bietet aber die Möglichkeit diese "Fehler" (Kleppin 1998) innerhalb der Unterrichtsmaterie zu behandeln und dem Lerner so zu helfen, Strategien zu entwickeln, um andere Textproduktionen kritisch beurteilen zu können.

Unser autlentisches Textmaterial in einer nicht deutschsprachigen Umgebung ist natürlich relativ "arm" und "defekt" aber trotzdem authentisch. Eine deutsche Speisekarte wäre in diesem Kontext logischerweise "nicht relevant" und völlig "unauthentisch".

Wichtig ist, dass die Schüler am Ende einer Unterrichtseinheit eigene Speisekarten produziert haben, die in gewisser Weise authentisch sind, weil sie in einem Restaurant verwendet werden können. Der Schüler geht mit einem fertigen Textprodukt nach Hause, das er oder sie bei der nächsten Gelegenheit am Arbeitsplatz im Restaurant sofort verwenden kann.

\section{Zur Wortschatzarbeit im Bereich Tourismus}

Das Ziel einer Unterrichtseinheit kann es sein, einen spezifischen Wortschatz (Müller 1994) der lokalen Gastronomie einzuführen, zu ordnen, mit anderen gastronomisch-kulturellen Kontexten zu vergleichen und Strategien zu vermitteln, wie dieses spezielle lokale Essen einem Fremden erklärt werden kann.

Zu Beginn wird eine Speisekarte, nur mit den Oberbegriffen und zusammen mit den authentischen Speisekarten aus der Region und einer Wortliste (Arbeitsblatt 1 und 2, siehe Anhang) verteilt. Den Schülern ist von inrem Beruf her die typische Einteilung einer Speisekarte bekannt. Trotzdem kann aber der Verständnisprozess mit Symbolen wie Fisch etc. unterstützt werden. Unter den Oberbegriffen können auch Prototypen (Müller 1994) eingesetzt werden: Nudelgerichte: Spaghetti als

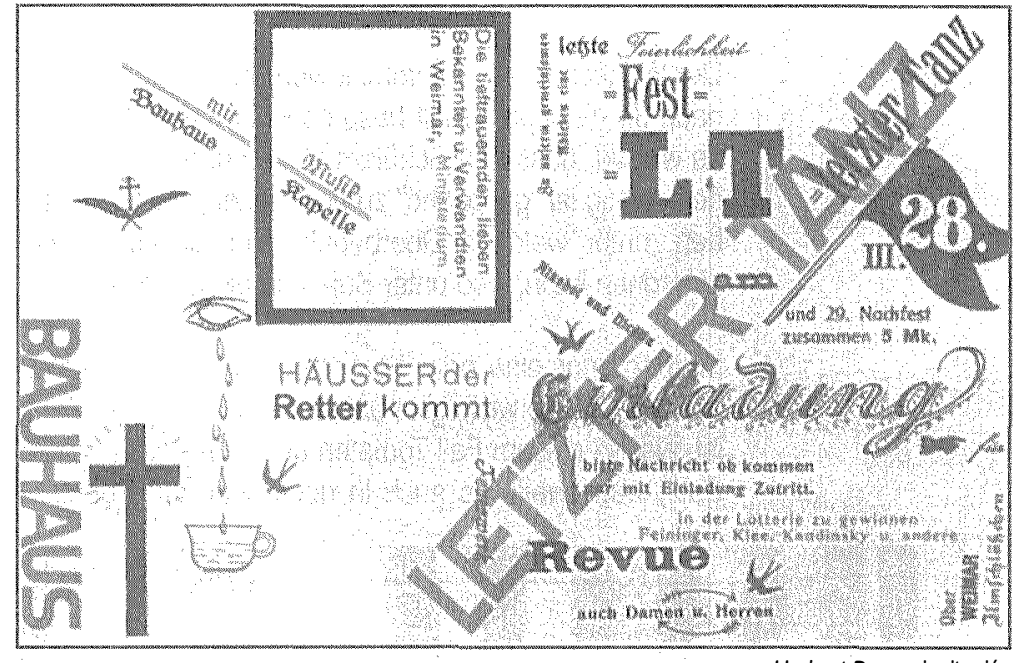

Prototyp, so verstehen sie das Wort Nudelgerichte, das sie noch nicht kennen. Darunter müssen die Schüler jetzt noch andere Nudelgerichte finden z. B. Makarroni

Herbert Bayer, invitacion para la última fiesta de la Bauhaus en Weimar, 1925 etc.

Suppe, Fleisch, Fisch ist bereits aus den Tagen zuvor bekannt. Die verschiedenen Tiere können dazu abgebildet werden. Rind und ein Rind daneben etc.

Die Schüler finden jetzt aus den Speisekarten die Gerichte und tragen es in inr Arbeits blatt oder in ihre Speisekarte ein

Bei Wortschatzarbeit für den spezifischen Fachbereich wird gerne gefragt, "wie heißt das auf deutsch?". Bei Fischgerichten stellt sich hier das Problem, dass ein Fisch einem Deutschen weder auf Spanisch noch auf Deutsch bekannt ist, wenn er diesen Fisch weder gesehen noch gegessen hat.

Wichtig ist hier, dass der Fisch beschrieben werden kann.

Woher kommt der Fisch?

Ist er groß oder klein?

Welche Farbe hat er?

Wie schmeckt er?

Wie isst man den Fisch? Gebraten, frittiert? Mit Knoblauch?

Mögliche Antworten: Der Fisch kommt aus der Bucht von Cádiz, heisst auch Salinenfisch, er schmeckt deswegen anders. Er schmeckt deswegen aber besonders gut.

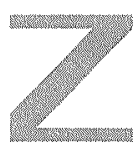

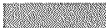


Der Wortschatz ist gesammelt worden und gemeinsam nach der Gruppenarbeit besprochen worden. Dabei ist es wichtig, dass den Schülern klar wird, dass es weniger wichtig ist "gazpacho" zu übersetzen, als zu verstehen, unter welchen Oberbegriff man dieses Gericht einordnen kann, also unter Suppe in diesem Fall.

Man soll nur eine oder höchstens zwei Hauptzutaten nennen, die die wichtigste bzw. die signifikanteste Zutat ist, also in diesem Fall Tomaten und nicht Wasser oder Salz, was zweifellos auch in der Suppe ist. Ebenfalls

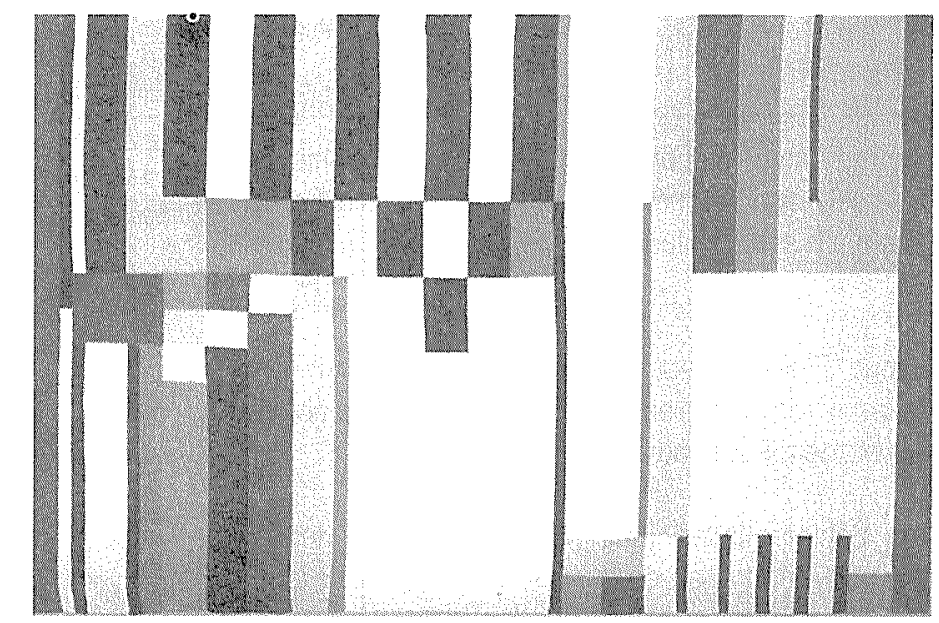

Ruth Hollos-Consemüller, tapiz, 1926

können besondere Kennzeichen hinzugefügt werden: Die Suppe ist kalt, nicht heiß.

\begin{tabular}{|l|}
\hline Gazpacho \\
\hline Oberbegriff: Suppe \\
\hline Hauptzutat: Tomaten \\
\hline Besondere Kennzeichen: kalt \\
\hline
\end{tabular}

Dieses System hilft, jegliche spezielle und regionale Speise in einer einfachen Form zu erklären.

Man kann nach verschiedenen Gerichten fragen und mit einfachen Redemitteln antworten:

"Was ist ein gazpacho?"

"Eine Suppe, mit Tomaten, sie ist kalt."

Mit dieser Art von Analyse gewinnen die Schüler Sicherheit, weil innen damit eine Strategie vermittelt wird, wie sie jegliche Art von Speise, auch wenn sie noch so kompliziert erscheint und keine Übersetzung dafür im Wörterbuch gefunden werden kann, erklären können?
Man kann dieses Schema auch abändern: An Stelle von "besondere Kennzeichen" kann man auch "Wie zubereitet?" fragen und mit "gebraten", "gegrillt" oder dergleichen antworten.

\section{Zur Textproduktion}

Als nächste Aufgabe sollen nun die Schüler wieder in Gruppen in unterschiedlicher Zusammensetzung selbst eine Karte produzieren. Die Speisekarten, die vorher ausgeteilt worden sind, werden eingesammelt. Die Schüler benützen aber ihre Arbeitsblätter und bei dieser Phase können sie auch Wörterbücher und ein spezielles gastronomisches Wörterbuch (Franco Cañero 1978) benützen.

Die Schüler benützen auch große Blätter, farbige Kartons und Buntstifte, um ihre persönliche Karte auch graphisch zu gestalten.

Aus der vorhergehenden Wortschatzarbeit wählen sie verschiedene Gerichte aus, die sie in ihrer speziellen Speisekarte gerne haben wollen. Sie entscheiden sich zum Beispiel entweder für ein Fisch- oder ein Fleischrestaurant. Sie schreiben die Oberbegriffe und darunter die entsprechenden Speisen und auch die Preise.

Die Anordnung könnte so sein:

Hauptgerichte:

Reisgerichte:

Paella (Reis mit Muscheln, Paprika etc.) ............1200,-

All diese Aktivitäten haben nur Sinn, wenn es zu den lokalen gastronomischen Bedingungen des Unterrichtsortes oder den Bedingungen der touristischen Region Bezug genommen wird. Der Lernerfolg hängt sehr stark von diesem relevanten Kontextbezug ab.

Kann diese Speisekarte in der Gegend des Unterrichtsortes in einem Restaurant ausliegen? Ist es möglich, dass ein Restaurant dies zu realistischen Preisen anbietet?

Dies sind Fragen, die nicht übergangen werden sollten. 


\section{Projekt- und Gruppenarbeit}

Eine andere mögliche Aktivität ist Projekt- und Gruppenarbeit: Zum Beispiel die Vorbereitung von Ausflügen. Tagesausflüge oder auch mehrtägige Ausflüge werden in Hotels oder in Reisebüros angeboten, um den Touristen, eine Abwechslung während ihres Aufenthaltes zu bieten. Es gibt innen die Möglichkeit, die Umgebung ihres Ferienortes kennenzulernen. Diese Aktivität ist für Schüler geeignet, die bereits zwischen 200 und 300 Unterrichsstuden hinter sich haben.

Verschiedene Gruppen der Klasse bereiten reelle oder mögliche reelleAusflüge vor. Sie suchen das Material, in Bibliotheken, Tourismusbüros, gestalten den Tagesplan, organisieren Eintrittskarten, Museumsbesuche, Pausen in Cafés oder Restaurants etc.

Die Lernziele dieser Projektarbeit sind unter anderen: - die Entwicklung der Fähigkeit der Lerner, eine touristische Aktivität für mögliche deutsch sprechende Reisende zu planen und zu organisieren,

- den Umgang mit modernen Kommunikationssystemen zu lernen, wie Internet, e-mail, fax und Telefon,

- die Entwicklung der Fähigkeit, Anträge zu stellen, Aufträge zu geben und Kostenvoranschläge zu erstellen - die Entwicklung der Fähigkeit, entsprechendes Material und Quellen zu suchen, zu beurteilen und anzuwenden

- die Entwicklung der Fähigkeit, mit verschiedenen Informationsquellen in verschiedenen Sprachen zu arbeiten - die Entwicklung der Fähigkeit, für die Zielreisegruppe einen adequaten Aktivitätsplan zu erstellen

- die Entwicklung der Fähigkeit, Aspekte der eigenen Kultur dem interessierten Fremden in der Sprache des Besuchers zu vermitteln

Die Schüler machen einen Prospekt, der verteilt wird. Auf dem Prospekt befindet sich der Termin des Ausfluges, Tagesablauf, Treffpunkt, Stadtplan etc. Der Ausflug wird dann abgehalten und der Rest der Klasse spielt Touristen. Es können aber auch Deutsche dazu eingeladen werden. Je nach Unterrichtsort sind nicht immer alle dazu nötigen Medien zur Verfügung oder es gibt Schwierigkeiten bei der Organisation des Projektunterrichts. Einer der Hauptaufgaben des Lehrers besteht darin, mit Schulleitungen zu verhandeln, damit Projektarbeit überhaupt realisiert werden kann. Z. B. die Möglichkeit das Büro, fax, Computer, Kopierer der Schule oder andere Einrichtungen zu benutzen, die ursprünglich nicht zu Unterrichtszwecken vorgesehen waren. Auch sollte bevor Projektunterricht begonnen wird, mit der Schulleitung verhandelt werden, ob sich Schüler während der Unterrichtszeit entfernen können oder ähnliche praktische Schwierigkeiten, die je nach institutionellem Kontext ganz verschieden sind.

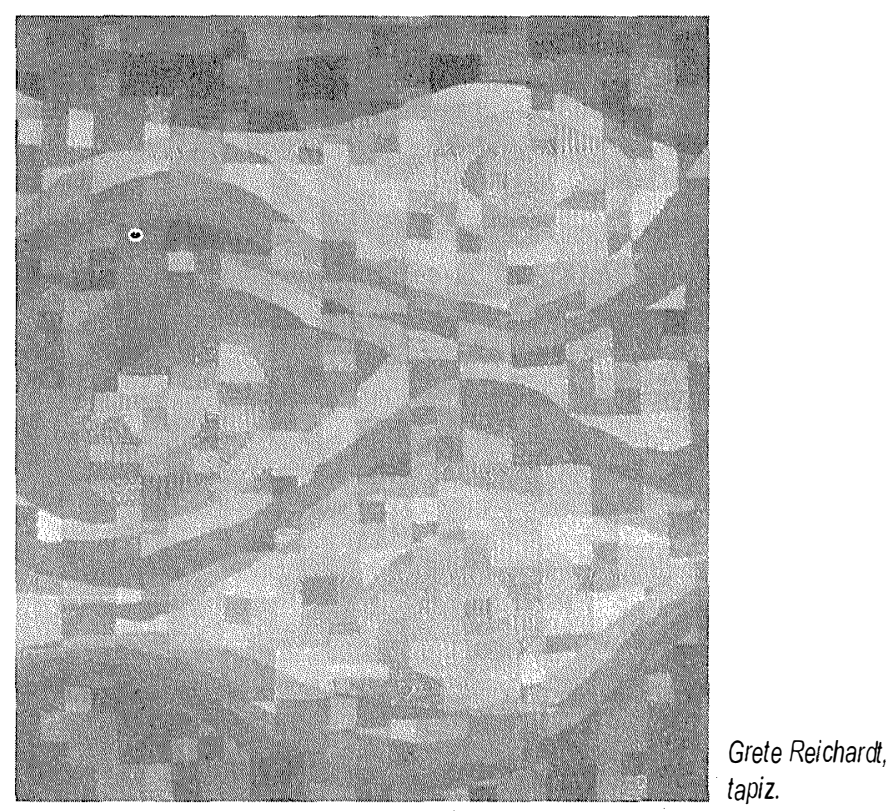

Eine andere wichtige Funktion des Lehrers ist es, die Gruppenarbeit zu koordinieren und zu unterstützen. Es kommt besonders darauf an, dass die Autonomie der Lernenden und die Eigeninitiativen nicht unterbrochen werden, da sie wichtige Elemente des Lernprozesses darstellen, aber andererseits sollen die Lerner auch nicht überfordert sein und sie sollen sich nicht vom Lehrer allein gelassen fühlen, da die Aufgabenstellung sehr umfangreich ist. Hilfestellungen sind wichtig, bei der Auswahl der Quellen, Entwicklung von Suchfragen, Vereinfachungsstrategien bei der Formulierung von Denkmälerbeschreibungen oder bei der Lösung von Problemen, die während des Arbeitsprozesses entstehen, zum Beispiel eine kurzfristige Änderung des Tagesplanes, weil eine Aktivität nicht möglich ist.

Es können Probleme entstehen, die man nicht voraussehen kann und die sowohl für die Lernenden, als auch für die Lehrer eine Herausforderung darstellen, dieses gemeinsam lösen zu können. All diese Prozesse tragen zu wirklichen Erfahrungsprozessen bei und ermöglichen so Lernerfolge in relevanten Kontexten.

R.S.
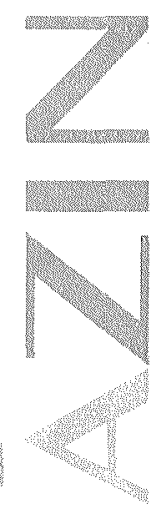


\section{Bibliographie:}

Albert Piñole, ISABel (1992), Gestión y Técnicas de Agencias de Viajes, 3ำ edición, Madrid

Bimmel, Peter/ Kast, Bernd/ Neuer, Gerd (1994), Arbeit mit Lehrwerkslektionen, München

Blanco Garcia, Nieves (1995), "La enseñanza secundaria obligatoria en una sociedad democrática" en: Fernández Sierra, Juan (ed.), El trabajo docente y psicopedagógico en educación secundaria, Málaga, pp. 17-42

Bausch, Karl-Richard/ Christ, Herbert/ Krumm, Hans-JürGEN, (Hrsg.) (1995), Handbuch Fremdsprachenunterricht, 3. Auflage, Tübingen

CONTRERAS Domingo, José (1994), "La investigación en la acción. ¿Que es?" ¿Como se hace?", en: Cuadernos de Pedagogía 224, pp. 8-19

Dahlhaus, Barbara (1994), Fertigkeit Hören, 1994

Freyer, Walter (1993), Tourismus, Einführung in die Fremdenverkehrsökonomie, 4. Auflage, München

Funk, Hermann/ Koenig, Michael (1996) Grammatik lehren und lernen; München, 4. Auflage

HAIDL, ANTON (1998), "La teoría de la relevancia y los procesos de adquisición en la enseñanza de idiomas extranjeros", Pragmática, Cádiz, pp. 367-78

- (1999), "Spracherwerb 'Revisited': Die ersten 50 Stunden Deutsch als Fremdsprache", in Akten des X. IDV-Kongresses, Amsterdam, 1998.

- "Interview mit L. Robledo - Präsident des Fachverbands des Cadizer Hotel- und Gaststättengewerbes", in: aga-magazin Nr. 4, 1998, S. 53-54

KLEPPIN, KarIN (1998), Fehler und Fehlerkorrektur, München

Linke, Angelika/ Nussbaumer, Markus/ Portmann, Paul (1991), Studienbuch Linguistik, Tübingen

LÜGER, HEINZ-HELMUT (1993), Routinen und Rituale in der Alltagskommunikation, Tübingen
Montaner Montejano, Jordi (1990), Estructura del Mercado Turístico, Madrid

MüLleR, BERnd-Dietrich (1994), Wortschatzarbeit und Bedeutungsvermittlung, Tübingen

Neuf-Münkel, Gabriele/ Roland, Regine (1995), Fertigkeit Sprechen, München

Neuner, Gerhard/ Hunfeld, Hans (1996), Methoden des fremdsprachlichen Deutschunterrichts, Kassel, 2. Auflage

Neuner, Gerhard/ Krüger, Michaed Grewer, UlRich (1994), Übungstypologie zum kommunikativen Deutschunterricht, 9. Auflage, Berlin/ München

Neuner, Gerhard (1998), "Gesellschaftspolitische Entwicklungen in Europa und inre Auswirkungen auf Deutsch als Fremdsprache" in: aga-magazin Nr. 4, S. 42-52

Pérez Gómez, Angel (1995), "Curriculum y escolaridad", en: Felix Angulo/ Nieves Blanco (ed), Teoría y desarollo del curriculum, Málaga, 67-76

SCHERER, BRIGITTE (1995), Tourismus, Hamburg

Scherling, Theo/ Schuckall, Hans-Friedrich (1996), Mit Bildern lernen, 4. Auflage

Westhof, Gerhard (1997), Fertigkeit Lesen, München

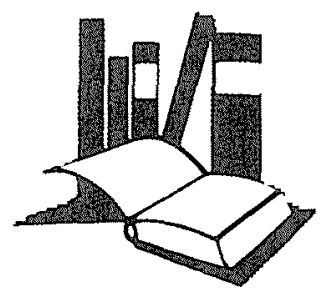

